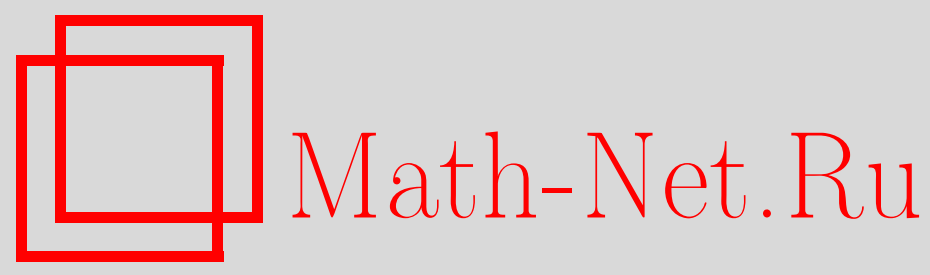

Ю. С. Шуткин, О реализации булевых функций информационными графами, Дискрет. матем., 2008, том 20, выпуск 4, 29-41

DOI: https://doi.org/10.4213/dm1024

Использование Общероссийского математического портала Math-Net.Ru подразумевает, что вы прочитали и согласны с пользовательским соглашением http://www . mathnet.ru/rus/agreement

Параметры загрузки:

IP: 54.162 .27 .143

26 апреля 2023 г., 12:30:54 
УДК 519.7

\title{
О реализации булевых функций информационными графами
}

\author{
() 2008 г. Ю. С. Шуткин
}

\begin{abstract}
Рассматривается задача реализации булевых функции с помощью информационных графов. Получено точное значение функции Шеннона сложности их реализации в классе древовидных информационных графов. Для почти всех булевых функций получен порядок сложности их реализации информационными графами и асимптотика сложности их реализации информационными деревьями.
\end{abstract}

\section{1. Введение}

Рассматривается задача реализации булевых функции с помощью информационных графов (понятие информационного графа введено в [1]). Такая реализация дает возможность, в зависимости от вероятностного пространства запросов, строить различные информационные графы для минимизации числа действий, затраченных на вычисление функции. В нашей задаче эти действия есть ни что иное, как вычисление предикатов на ребрах графа, а суммарно затраченные усилия - сложность этого графа, причем сложность можно считать как на одном конкретном запросе, так и в среднем, что дает наиболее четкое представление о том, как минимизировать число действий в зависимости от заданных вероятностей запросов.

Реализация функций с помощью информационных графов может пригодиться, например, когда мы эмулируем контактную схему и ее функционирование на компьютере. В этом случае функционал сложности для простейших информационных графов как раз отвечает затратам на вычисление предикатов на контактах схемы.

Когда это понятно из контекста, мы будем вместо термина информационный граф использовать термин граф.

Построить какой-то информационный граф, реализующий заданную функцию, не составляет труда. Достаточно рассмотреть совершенную дизъюнктивную нормальную форму (СДНФ) функции. Понятно, что последовательность идущих друг за другом ребер реализует конъюнкцию, а пучок ребер, выходящих из одной вершины - дизъюнкцию. Поэтому строим несколько путей, реализующих каждую конъюнкцию в СДНФ, из начальной вершины в конечную. Граф будет реализовывать нашу функцию. Отсюда легко можно получить и верхнюю оценку сложности задачи, так как число конъюнкций в СДНФ очевидным образом зависит от $n$, а длина каждой из них конечна, и сложность легко вычисляется.

Представляет интерес отдельно рассматривать задачу в классе информационных деревьев. Деревья, как правило, могут быть реализованы проще, поэтому их нельзя полностью заменить графами. 
Получены основные оценки сложности реализации, такие, как оценка функции Шеннона сложности реализации функции в классе информационных графов и информационных деревьев (для деревьев она является точной). Также для почти всех булевых функций получен порядок сложности реализации их информационными графами, а для деревьев установлена асимптотика сложности.

Результаты данной статьи анонсированы в [2].

Автор благодарен Э. Э. Гасанову за постановку задачи и помощь в исследовании.

\section{2. Постановка задачи и формулировка результатов}

Информационным графом $G$ с базовым множеством $F=\left\{x_{1}, \bar{x}_{1}, x_{2}, \bar{x}_{2}, \ldots, x_{n}, \bar{x}_{n}\right\}$ будем называть сеть с несколькими выделенными вершинами (одна начальная и несколько конечных), в которой все ребра ориентированы от начальной вершины к конечным и ребрам приписаны предикаты из множества $F$ (по сути, если отождествить все конечные вершины, то определение совпадает с определением ориентированной контактной схемы [3], с той лишь разницей, что сложность графа задается по-другому).

Считается, что ребро с предикатом $x_{i}^{\sigma}$ проводит запрос $\alpha=\left(\alpha_{1}, \alpha_{2}, \ldots, \alpha_{n}\right) \in\{0,1\}^{n}$ тогда и только тогда, когда $\alpha_{i}=\sigma$.

Говорим, что запрос $\alpha \in\{0,1\}^{n}$ проходит из вершины $v_{1}$ в вершину $v_{2}$ (или вершина $v_{2}$ достижима из $v_{1}$ на запросе $\alpha$ ), если существует ориентированный путь из $v_{1}$ в $v_{2}$ такой, что все ребра этого пути проводят запрос $\alpha$.

Обозначим множество вершин, достижимых из $v_{1}$ на запросе $\alpha$, через $\theta_{v_{1}}(\alpha)$. Множество конечных вершин $w_{j}$ обозначим через $W$. Начальную вершину будем обозначать $v_{0}$.

Для информационного дерева потребуем, чтобы все конечные вершины были листьями дерева. Высотой дерева будем считать длину максимального пути из начальной вершины в конечную.

Информационный граф $G$ реализует булеву функцию $f\left(x_{1}, x_{2}, \ldots, x_{n}\right)$, если любой набор $\alpha \in\{0,1\}^{n}$, на котором функция принимает значение 1 , проходит из начальной вершины графа $G$ в одну из конечных вершин, то есть $\theta_{v_{0}}(\alpha) \cap W \neq \varnothing$, и любой набор $\beta \in\{0,1\}^{n}$, на котором функция принимает значение 0 , не проходит ни в одну из них, то есть $\theta_{v_{0}}(\alpha) \cap W=\varnothing$. Множество графов, реализующих функцию $f$, обозначим через $U(f)$.

Более общее определение информационного графа и его функционирования можно найти в [1].

Сложностью графа на запросе $\alpha$ назовем число предикатов, вычисленных на этом запросе.

Число предикатов, вычисленных на запросе $\alpha$ в графе $G$, подсчитывается следующим образом. Помечаются все вершины, в которые проходит запрос $\alpha$. Считаем, что в вершине $v$ вычисляются те предикаты, которые приписаны ребрам, выходящим из этой вершины. Общее число вычисленных предикатов на запросе $\alpha$ равно сумме по всем помеченным вершинам вычисленных в них предикатов, то есть

$$
L(G, \alpha)=\sum_{v \in \theta_{v_{0}}(\alpha)} \psi(v),
$$

где $\psi(v)$ - число ребер, выходящих из $v$ (степень исхода вершины $v$ ). 
Пусть на множестве запросов введено вероятностное пространство. Сложностью информационного графа назовем величину

$$
L(G)=\sum_{\alpha \in\{0,1\}^{n}} L(G, \alpha) P(\alpha)=\mathbf{E}_{\alpha}(L(G, \alpha)),
$$

где $P(\alpha)$ - вероятность запроса $\alpha$ в вероятностном пространстве, а $\mathbf{E}_{\alpha}(L(G, \alpha))$ - среднее значение $L(G, \alpha)$. Далее будем по умолчанию считать, что распределение равномерное, то есть вероятности появления всех запросов равны.

Сложностью $L(f)$ функции $f$ назовем нижнюю грань сложности графов, реализующих эту функцию:

$$
L(f)=\inf _{G \in U(f)} L(G) .
$$

Через $P_{2}^{(n)}$ обозначим множество всех булевых функций, зависящих от переменных $x_{1}, \ldots, x_{n}$. Функцией Шеннона $L^{S h}(n)$ сложности реализации булевых функций $n$ переменных информационными графами назовем максимальную сложность функций из $P_{2}^{(n)}$, то есть

$$
L^{S h}(n)=\max _{f \in P_{2}^{(n)}} L(f) .
$$

Определим также сложность реализации булевой функции с помощью информационных деревьев и функцию Шеннона для древовидных информационных графов.

Сложность реализации функции деревом определим как

$$
L_{D}(f)=\inf _{G \in D(f)} L(G)
$$

где $D(f)$ - множество деревьев, реализующих функцию $f$. На самом деле мы увидим, что нижняя грань достигается, то есть в этом определении и в определении сложности реализации функции информационными графами можно заменить inf на min.

Функцию Шеннона в классе деревьев определим как

$$
L_{D}^{S h}(n)=\max _{f \in P_{2}^{(n)}} L_{D}(f) .
$$

Итак, ставится задача построения графа с минимальной сложностью.

Получена верхняя оценка сложности реализации функции $n$ переменных в классе информационных графов. А именно, для любой функции $f$ из $P_{2}^{(n)}$ справедливо неравенство

$$
L(f) \leqslant 2 n-1 .
$$

В классе деревьев эта оценка совпадает с нижней оценкой функции Шеннона, то есть

$$
L_{D}^{S h}(n)=2 n-1 .
$$

В классе графов получена более слабая нижняя оценка функции Шеннона

$$
L^{S h}(n) \geqslant \frac{3 n-1}{2} \text {. }
$$

Найден порядок сложности для почти всех функций $f \in P_{2}^{(n)}$ их реализации с помощью информационных графов, $L(f) \asymp n$, и асимптотика сложности реализации информационными деревьями для почти всех функций $f \in P_{2}^{(n)}, L_{D}(f) \sim 2 n$. 


\section{3. Верхняя оценка}

Лемма 1. $B U(f)$ сущеествует информациионный граф, на котором достигается сложность функции $f$.

Доказательство. Рассмотрим произвольный граф из $U(f)$. Поставим каждой вершине этого графа в соответствие множество запросов, которые туда проходят. Из определения сложности легко видеть, что две вершины, которым сопоставлены одинаковые множества запросов, можно отождествить (склеить) без потери функциональности и без изменения сложности. Будем называть графы, полученные друг из друга с помощью такого преобразования, эквивалентными.

Таким образом, среди бесконечного числа информационных графов можно выделить конечное множество (по одному из каждого класса эквивалентности), а в нем уже найти граф с минимальной сложностью. На нем и будет достигаться сложность функции. Лемма доказана.

Получим верхнюю оценку сложности.

Лемма 2. Для любой функции п переменных $f$ из класса $P_{2}^{(n)}$

$$
L(f) \leqslant 2 n-1 .
$$

Доказательство. Построим граф, реализующий функцию, следующим образом (приведенный метод схож с методом каскадов для контактных схем, см. [3]). Из корня выходит два ребра $x_{1}$ и $\bar{x}_{1}$. Дальше на концах этих двух ребер строим графы, реализующие соответственно функции $f\left(1, x_{2}, \ldots, x_{n}\right)$ и $f\left(0, x_{2}, \ldots, x_{n}\right)$. Получим, что весь граф реализует функцию

$$
x_{1} f\left(1, x_{2}, \ldots, x_{n}\right) \vee \bar{x}_{1} f\left(0, x_{2}, \ldots, x_{n}\right)=f\left(x_{1}, x_{2}, \ldots, x_{n}\right) .
$$

Продолжаем такое построение, пока не дойдем до функции одной переменной. Ее реализуем одним ребром (или нулем ребер, если это константа).

Пусть $G^{(n)}-$ граф, реализующий заданную функцию $n$ переменных таким способом. Тогда $L\left(G^{(n)}\right) \leqslant 2 n-1$. Докажем это индукцией по числу переменных. На функцию одной переменной надо не больше одного ребра, следовательно, $L\left(G^{(1)}\right) \leqslant 1$. Используя разложение по первой переменной, реализуем функцию $n$ переменных способом, описанным выше. Вероятность того, что запрос пройдет по ребру, выходящему из корня, равна 1/2, и дальше ребер с таким предикатом мы не встретим, следовательно, вероятности всех ребер графа $G_{1}$ (того, который построен на левом ребре, выходящем из корня) уменьшатся вдвое. То же самое для $G_{2}$. В итоге получим, что

$$
L\left(G^{(n)}\right)=2+\frac{1}{2} L\left(G_{1}^{(n-1)}\right)+\frac{1}{2} L\left(G_{2}^{(n-1)}\right) .
$$

Отсюда по предположению индукции получаем, что

$$
L\left(G^{(n)}\right) \leqslant 2+\frac{1}{2}(2(n-1)-1+2(n-1)-1)=2 n-1 .
$$

Итак, сложность построенного графа не превышает $2 n-1$. Это означает, что сложность задачи реализации функции $n$ переменных удовлетворяет неравенству $L(f) \leqslant 2 n-1$. Таким образом, получена верхняя оценка и лемма доказана.

Заметим, что если распределение не является равномерным, данная верхняя оценка сложности останется верной, так как существенно здесь используется только тот факт, что сумма вероятностей того, что запрос пройдет по $x_{i}$ и по $\bar{x}_{i}$, равна единице, а это выполнено всегда. 


\section{4. Нижняя оценка функции Шеннона в классе деревьев}

Докажем лемму о нижней оценке для реализации функции информационными деревьями.

Лемма 3. Функияя Шеннона сложности реализации функции $n$ переменных из класса $P_{2}^{(n)}$ с помощьюю информационных деревьев удовлетворяет неравенству

$$
L_{D}^{S h}(n) \geqslant 2 n-1 .
$$

Доказательство. Покажем, что с помощью деревьев реализовать функцию $f=\bigoplus_{i=1}^{n} x_{i}$ со сложностью, меньшей $2 n-1$, не удастся. Пусть существует дерево $D$, реализующее эту функцию. Рассмотрим все пути, по которым может пройти хоть какой-нибудь запрос $x$ из начальной вершины в конечную. Это в точности такие пути, на ребрах которых любая переменная $x_{i}$ встречается либо везде с отрицанием, либо везде без него. Остальные пути можно просто исключить (то есть удалить ребра, не принадлежащие ни одному из указанных выше путей), это не увеличит сложность дерева, и не изменит его функциональности.

Покажем, что любой путь этого дерева из начальной вершины в конечную не меньше $n$. Предположим противное: пусть существует путь из начальной в конечную вершину, длина которого меньше $n$. Тогда среди предикатов на ребрах этого пути отсутствует какая-либо переменная, то есть существует $j$ такое, что нет ни $x_{j}$, ни $\bar{x}_{j}$. Тогда возьмем произвольный запрос $x$, который проходит по этому пути. Дерево реализует функцию $f=\bigoplus_{i=1}^{n} x_{i}$, поэтому $\bigoplus_{i=1}^{n} x_{i}=1$ на этом запросе. Но на запросе $x^{\prime}=x \oplus e_{j}$, где $e_{j}=(0, \ldots, 0,1,0, \ldots, 0)-$ единичный базисный вектор, эта функция равна нулю, несмотря на то, что запрос $x^{\prime}$ тоже пройдет в конечную вершину по тому же пути, что и запрос $x$, так как в этом пути нет предиката $x_{j}$. Пришли к противоречию.

Теперь укоротим дерево до высоты $n$ следующим образом. Возьмем произвольный путь и предположим, что его длина больше $n$. Тогда в этом пути существуют два ребра $d_{1}$ и $d_{2}$ (упорядоченные от корня к конечной вершине), которым приписан один и тот же предикат (либо $x_{i}$, либо $\bar{x}_{i}$ ). Второе ребро можно удалить, при этом сложность понизится ровно на $P(v)$, где $v$ - вершина, из которой выходило ребро $d_{2}$, а функциональность не изменится, так как все запросы, которые проходили через $d_{2}$, проходили и через $d_{1}$ (так как это дерево), следовательно, мы лишний раз проверяли условие, которое и так выполнялось.

Таким образом, получили, что все пути оптимального дерева, реализующего функцию $\bigoplus_{i=1}^{n} x_{i}$, из начальной вершины в конечные имеют длину $n$.

Более того, если существуют одинаковые пути, их можно удалить, оставив только один (одинаковые с точностью до перестановки ребер, то есть пропускающие одинаковые запросы). После такого преобразования останется ровно $2^{n-1}$ различных путей, каждый отвечающий своей единице функции $f$. Таким образом, число листьев дерева (конечных вершин) равно $2^{n-1}$. Заметим, что граф (который является деревом), построенный по принципу, описанному в разделе 3 , для функции $f$ имеет на предпоследнем $(n-1)$-м ярусе $2^{n-1}$ вершин, на $(n-2)-\mathrm{M}-2^{n-2}$ вершин, и так далее, на нулевом имеется одна вершина (корень).

Вернемся к оптимальному дереву. Предположим, что на каком-то $i$-м ярусе, $i<n$, этого дерева вершин меньше, чем $2^{i}$, значит, из какой-то вершины $v$ на этом ярусе растет поддерево высоты $n-i$, у которого конечных вершин больше, чем $2^{n-i-1}$. Но так как к вершине $v$ ведет единственный путь из корня длины $i$, значит, $i$ переменных уже задействованы, и осталось только $n-i$ переменных (мы знаем, что каждая переменная присутствует, но длина равна $n$, следовательно, каждая из переменных присутствует по 
одному разу). Без ограничения общности, можно считать, что задействованы $x_{1}, \ldots, x_{i}$, а остались $x_{i+1}, \ldots, x_{n}$. Таким образом, получаем, что в вершине $v$ растет дерево, которое реализует функцию $n-i$ переменных $f^{\prime}$, у которой больше $2^{n-i-1}$ единиц, то есть больше, чем число единиц функции $\left.f\right|_{n-i+1, \ldots, n}=\bigoplus_{j=n-i+1}^{n} x_{j}$. Функция $f^{\prime}$ больше чем на половине наборов принимает значение 1 , следовательно, существуют два соседних набора $\alpha$ и $\alpha^{\prime}$ (отличающихся только в одной позиции), на которых эта функция принимает значение 1 , следовательно, существуют два пути из $v$ в конечные вершины, которые проводят запросы $\alpha$ и $\alpha^{\prime}$. Вместе с ними существуют два соседних набора $\beta$ и $\beta^{\prime}$ длины $n$, полученные дополнением из $\alpha$ и $\alpha^{\prime}$ так, чтобы запросы $\beta$ и $\beta^{\prime}$ проходили в вершину $v$, и существуют два пути из начальной вершины в конечные, которые проводят эти запросы. Отсюда следует, что значение $f$ на этих наборах равно единице. Это противоречит тому, что дерево реализовывало исходную функцию $f=\bigoplus_{j=1}^{n} x_{j}$.

Итак, на каждом $i$-м ярусе не меньше $2^{i}$ вершин, где $i=0,1, \ldots, n-1$, а на последнем $n$-м ярусе $2^{n-1}$ вершин, в точности столько, сколько единиц у функции $f$. Таким образом, число ребер, выходящих с $i$-го яруса равно числу вершин на следующем, и значит, не меньше $2^{i+1}, i=0,1, \ldots, n-2$, и не меньше $2^{n-1}$ ребер, выходящих с предпоследнего яруса. Вероятность прохождения запроса в любую вершину $i$-го яруса равна $2^{-i}$.

Суммируя по всем вершинам, получаем, что

$$
L(D) \geqslant \sum_{i=0}^{n-2} 2^{i+1} 2^{-i}+2^{n-1} 2^{-n+1}=2(n-1)+1=2 n-1 .
$$

Таким образом, лемма доказана.

\section{5. Нижняя оценка функции Шеннона в классе информационных графов}

Получим теперь нижнюю оценку функции Шеннона $L^{S h}(n)$ для графов.

Лемма 4. Функичия Шеннона сложности реализации функциии $n$ переменных из класса $P_{2}^{(n)}$ с помощьью информационньхх графов удовлетворяет неравенству

$$
L^{S h}(n) \geqslant \frac{3 n-1}{2} \text {. }
$$

Доказательство. Пусть существует граф, реализующий функцию $f=\bigoplus_{i=1}^{n} x_{i}$, и пусть $n \geqslant 2$, в противном случае утверждение очевидно.

Покажем, что на каждом запросе $x$, на котором значение функции равно 1 , мы вычислим как минимум $2 n-1$ предикатов.

Пусть это не так, то есть существует запрос $\alpha=\left(\alpha_{1}, \alpha_{2}, \ldots, \alpha_{n}\right)$ такой, что $f(\alpha)=1$, и какие-то два предиката из $x_{1}, \bar{x}_{1}, x_{2}, \bar{x}_{2}, \ldots, x_{n}, \bar{x}_{n}$ не вычислены. Если это $x_{i}$ и $\bar{x}_{i}$, то приходим к противоречию, как в случае с деревьями. Пусть это $x_{i}^{\sigma_{i}}$ и $x_{j}^{\sigma_{j}}, i \neq j$. Без ограничения общности положим, что это $\bar{x}_{i}$ и $\bar{x}_{j}$. Так как каждый путь из корня в конечную вершину содержит все переменные, и по какому-то пути мы прошли, следовательно, прошли и по $x_{i}, x_{j}$, а в запросе $\alpha$, таким образом, $\alpha_{i}=1, \alpha_{j}=1$. Теперь рассмотрим запрос $\alpha^{\prime}$ такой, что $\alpha_{k}^{\prime}=\alpha_{k}, k \neq i, j$ и $\alpha_{i}^{\prime}=\alpha_{i} \oplus 1=0, \alpha_{j}^{\prime}=\alpha_{j} \oplus 1=0$. Значение функции на таком запросе равно единице, так как $\alpha^{\prime}=\alpha \oplus e_{i} \oplus e_{j}, f\left(\alpha^{\prime}\right)=f(\alpha) \oplus 1 \oplus 1=f(\alpha)=1$. Поэтому в графе должен существовать путь, по которому запрос $\alpha^{\prime}$ пройдет из корня в 
конечную вершину. Но такого пути не существует, так как все пути, по которым проходил запрос $\alpha$, перекрылись в силу того, что в них есть $x_{i}$ и $x_{j}$, а новых путей появиться не могло, так как мы не вычисляли ни одного ребра $\bar{x}_{i}$ или $\bar{x}_{j}$, которые могли бы открыть нам новый путь. Таким образом, приходим к противоречию, и минимальное число ребер, вычисленное на запросе $\alpha$, равно $2 n-1$. На остальных запросах $\tilde{\alpha}$ таких, что $f(\tilde{\alpha})=0$, вычисляем как минимум $n$ предикатов на ребрах. Предположим обратное, то есть предположим, что существует запрос $\tilde{\alpha}$, на котором мы вычислили меньше $n$ ребер, и $f(\tilde{\alpha})=0$. Значит, какая-то переменная осталась нетронутой, и, изменив эту переменную в запросе, мы должны пройти в конечную вершину, так как значение функции уже равно единице, но, так как эту переменную мы не трогали на запросе $\tilde{\alpha}$, никаких других путей на новом запросе не появится, и пройти туда мы не сможем. Пришли к противоречию. Так как таких запросов ровно половина, получаем, что сложность графа не меньше $2^{n-1}(2 n-1) 2^{-n}+2^{n-1} n 2^{-n}=(3 n-1) / 2$.

Таким образом, получена нижняя оценка функции Шеннона сложности реализации функций информационными графами, что и завершает доказательство леммы.

Теперь можно сформулировать теорему об оценках сложности реализации функций $n$ переменных.

Теорема 1. Для функции Шеннона сложности реализации функции п переменньх из класса $P_{2}^{(n)}$ с помощьью информационньх графов справедливы неравенства

$$
\frac{3 n-1}{2} \leqslant L^{S h}(n) \leqslant 2 n-1 .
$$

Для функция Шеннона сложности реализации функции $n$ переменных из класса $P_{2}^{(n)} c$ помощьюю информационных деревьев справедливо равенство

$$
L_{D}^{S h}(n)=2 n-1 .
$$

Утверждения теоремы следуют из лемм 2, 3, 4 .

\section{6. Порядок сложности для почти всех булевых функций}

Оценка функции Шеннона для графов получена в разделе 5. Теперь найдем порядок сложности реализации информационными графами для почти всех булевых функций.

Для этого выделим некоторый класс функций, для которых может быть получена простая оценка, и покажем, что на самом деле это почти все функции, то есть при $n \rightarrow \infty$ число остальных функций есть $o\left(2^{2^{n}}\right)$.

Введем на множестве всех функций две функции $\zeta_{0}(f)$ и $\zeta_{1}(f)$, принимающие значения из множества неотрицательных целых чисел.

Функция $\zeta_{h}(f)=k$ тогда и только тогда, когда существует набор индексов $i_{1}, \ldots, i_{k}$ и набор $\alpha_{i_{1}}, \ldots, \alpha_{i_{k}}$ такой, что $f\left(*, \ldots, *, \alpha_{i_{1}}, *, \ldots, *, \alpha_{i_{k}}, *, \ldots, *\right)=h$, другими словами, это означает, что при подстановке в функцию вместо $k$ переменных значений $\alpha_{i_{j}}$ получается подфункция $n-k$ переменных, которая есть тождественная константа $h$. Причем для $l=k-1$ такого набора $\alpha_{i_{1}}, \ldots, \alpha_{i_{l}}$ не существует, то есть это минимальное $k$, при котором выполнено это свойство.

Заметим, что для константных функций 0 и 1 соответствующие значения $\zeta_{1}$ и $\zeta_{0}$ не определены, но это не должно нас смущать, так как такие функции всегда очень легко рассматриваются отдельно. 
Дадим некоторую геометрическую интерпретацию введенным функциям. Выясним, что означает, что функция обращается в ноль при фиксировании нескольких переменных. Рассмотрим $n$-мерный булев куб, в узлах которого стоят значения функции на соответствующих наборах. Тогда фиксация переменной означает выбор половины исходного куба, фиксация нескольких переменных - выбор подкуба меньшей размерности, который соответствует подфункции, полученной из исходной подстановкой этих переменных.

Итак, число $\zeta_{0}(f)$ характеризует максимальную размерность подкуба, состоящего из одних нулей, то есть в кубе функции $f$ существует подкуб из одних нулей размерности $n-\zeta_{0}(f)$ и не существует нулевого подкуба большей размерности.

Аналогичным свойством обладает и функция $\zeta_{1}(a)$ и подкубы, состоящие из одних единиц.

Еще одна наглядная интерпретация связана со строением графов, которые реализуют данные функции.

Функция $\zeta_{1}(f)$ есть не что иное как минимально возможная длина пути, идущего из начальной вершины в конечную (имеются в виду пути, которые не содержат противоречивых предикатов, например $x_{i}$ и $\bar{x}_{i}$ одновременно).

Функция $\zeta_{0}(f)$, в свою очередь, накладывает ограничение на размер сечения в графе, разделяющего начальную вершину и множество конечных вершин, размер такого сечения не может быть меньше $\zeta_{0}(f)$. Опять же имеются в виду те сечения, которые могут перегородить какой-нибудь запрос (те, которые содержат $x_{i}$ и $\bar{x}_{i}$ одновременно, не подходят).

Доказательство этих фактов приведено в лемме ниже.

Обозначим через $\zeta(f)$ минимальную из $\zeta_{0}(f)$ и $\zeta_{1}(f)$ и назовем значение этой функции степенью существенности функции $f$.

Лемма 5. Для произвольной функции п переменных справедливы неравенства

$$
L(f) \geqslant 2^{-n}\left(\zeta_{0}(f) N_{0}(f)+\zeta_{1}(f) N_{1}(f)\right),
$$

где $N_{0}\left(N_{1}\right)$ - соответственно число нулей (единиц) функиии, и

$$
L(f) \geqslant \zeta(f) .
$$

Доказательство. Оценим сложность графа, реализующего произвольную функцию.

Пусть фиксирована функция $n$ переменных $f$ и построен граф, который ее реализует. Рассмотрим произвольный набор $\alpha$, на котором функция принимает значение 1 . По определению, существует путь в графе из начальной вершины в конечную, который проводит этот запрос. Предположим, что длина этого пути строго меньше, чем $\zeta_{1}(f)$. Тогда возьмем множество предикатов на ребрах этого пути $S=\left\{x_{i_{1}}^{\sigma_{i_{1}}}, x_{i_{2}}^{\sigma_{i_{2}}}, \ldots, x_{i_{s}}^{\sigma_{i_{s}}}\right\}, s<\zeta_{1}(f)$, и рассмотрим набор $\tilde{\gamma}=\left(\gamma_{i_{1}}=\sigma_{i_{1}}, \gamma_{i_{2}}=\sigma_{i_{2}}, \ldots, \gamma_{i_{s}}=\sigma_{i_{s}}\right)$. Все $i_{j}$ можно считать различными, так как если в наборе предикатов $S$ и встретились одинаковые переменные, то они встретились в одинаковой степени, иначе бы запрос не прошел, и, следовательно, можно оставить только по одному экземпляру, а остальные просто удалить. Мощность множества только уменьшится. Без ограничения общности считаем, что $i_{1}<i_{2}<\ldots<i_{s}$.

Легко видеть, что если мы произвольным образом дополним набор $\tilde{\gamma}$ до полного набора $\gamma=\left(\gamma_{1}, \ldots, \gamma_{i_{1}}, \ldots, \gamma_{i_{s}}, \ldots \gamma_{n}\right)$, то запрос $\gamma$ пройдет в конечную вершину, а значит, значение функции на нем равно 1. Это означает, что функция превратилась в тождественную единицу после подстановки в нее $\tilde{\gamma}$. Получаем противоречие с тем, что $\zeta_{1}(f)-$ минимальное число, обладающее таким свойством, а $s<\zeta_{1}(f)$.

Теперь рассмотрим произвольный набор $\alpha$, на котором функция принимает значение 0 . Предположим, что на этом наборе мы вычислили только $s$ предикатов на ребрах и так 
получилось, что $s<\zeta_{0}(f)$. Обозначим $S=\left\{x_{i_{1}}^{\sigma_{i_{1}}}, x_{i_{2}}^{\sigma_{i_{2}}}, \ldots x_{i_{s}}^{\sigma_{i_{s}}}\right\}$ множество вычисленных предикатов. Опять зафиксируем набор $\tilde{\gamma}$ длины $r$, который есть ограничение набора $\alpha$ на множество переменных, присутствующих в $S$. Длина набора $r$ может быть меньше $s$, так как какая-то переменная может встречаться в $S$ два раза (с отрицанием и без него). Получаем набор длины $r \leqslant s<\zeta_{0}(f)$. Нетрудно понять, что как бы мы теперь ни дополняли его до полного набора, то есть как бы ни фиксировали остальные переменные, полученный запрос будет проходить в точности в те же вершины графа, в которые уже прошел урезанный набор, так как ни одного доступного ребра с пометкой не из $S$ нет. Все это означает, что функция превратилась в константу 0. В итоге пришли к противоречию, опять нарушена минимальность $\zeta_{0}(f)$.

Итак, на любом наборе $\alpha$ таком, что $f(\alpha)=1$, существует путь длины, не меньшей $\zeta_{1}(f)$, по которому проходит запрос $\alpha$, а значит, мы вычислим хотя бы $\zeta_{1}(f)$ предикатов. Если же значение функции равно нулю, то необходимое число предикатов, которое должно быть вычислено, равно $\zeta_{0}(f)$. Отсюда следует, что сложность такой функции удовлетворяет неравенству

$$
L(f) \geqslant 2^{-n}\left(\zeta_{0}(f) N_{0}(f)+\zeta_{1}(f) N_{1}(f)\right) .
$$

Так как $\zeta(f)$ есть минимальная из $\zeta_{0}(f)$ и $\zeta_{1}(f)$, это неравенство можно переписать в несколько ослабленном виде $L(f) \geqslant \zeta(f)$.

Понятно, что функция $\zeta(f)$ характеризует минимальную длину набора, при подстановке которого функция превращается в константу (в ноль или единицу).

Доказательство леммы завершено.

Замечание 1. Из данной оценки, в частности, следует оценка функции Шеннона сложности реализации функции с помощью информационных деревьев, доказанная в лемме 3 .

Лемма 6. Число функиий, для которых выполнено условие $\zeta(f)>s$, не меньще, чем $2^{2^{n}}-2^{2^{n}-2^{n-s}+s+1}\left(\begin{array}{l}n \\ s\end{array}\right)$.

Доказательство. Оценим число функций, для которых значение $\zeta(f)$ не больше какогото фиксированного числа $s$. Неравенство $\zeta(f) \leqslant s$ означает, что существует набор $\alpha_{f}$ длины $s$ такой, что $f\left(*, \ldots, *, \alpha_{f i_{1}}, *, \ldots, *, \alpha_{f i_{s}}, *, \ldots, *\right)$ есть константа. Поставим его в соответствие функции: $f \leftrightarrow \alpha_{f}$.

Посмотрим, сколько различных функций может быть сопоставлено таким образом одному набору $\alpha$, то есть нужно рассмотреть $2^{n}$ наборов $\gamma^{l}, l=1,2, \ldots, 2^{n}$, длины $n$, и понять, сколькими способами может быть на них определена функция. Если $\gamma^{l}$ содержит в себе $\alpha$, то есть $\left(\gamma_{i_{1}}^{l}, \ldots, \gamma_{i_{s}}^{l}\right)=\left(\alpha_{i_{1}}, \ldots, \alpha_{i_{s}}\right)$, то функция уже фиксирована (либо ноль, либо единица), какими бы ни были остальные $\gamma_{j}^{l}$. Имеем два способа. Рассмотрим остальные наборы такие, что $\left(\gamma_{i_{1}}^{l}, \ldots, \gamma_{i_{s}}^{l}\right) \neq\left(\alpha_{i_{1}}, \ldots, \alpha_{i_{s}}\right)$. Всего их $2^{n}-2^{n-s}$ (так как наборов c $s$ фиксированными переменными $2^{n-s}$ ). Соответственно, на них можно фиксировать функцию произвольным способом. (На самом деле, далеко не произвольным, так как то, что функция обращается в константу после фиксирования одного множества переменных не значит, что она не обратится в другую или такую же константу, если фиксировать другое множество переменных. Но мы оцениваем число функций сверху и можем себе позволить немного загрубить эту оценку.)

Итак, число способов задать на этих наборах функцию не больше $2^{2^{n}-2^{n-s}}$.

Таким образом, всего функций, сопоставленных набору $\alpha$ длины $s$, не превосходит $2^{2^{n}-2^{n-s}+1}$ (остается еще двумя способами задать функции на наборах, содержащих $\alpha$ ). Число таких наборов равно $2^{s}$. А так как любой функции, для которой $\zeta(f) \leqslant s$, можно 


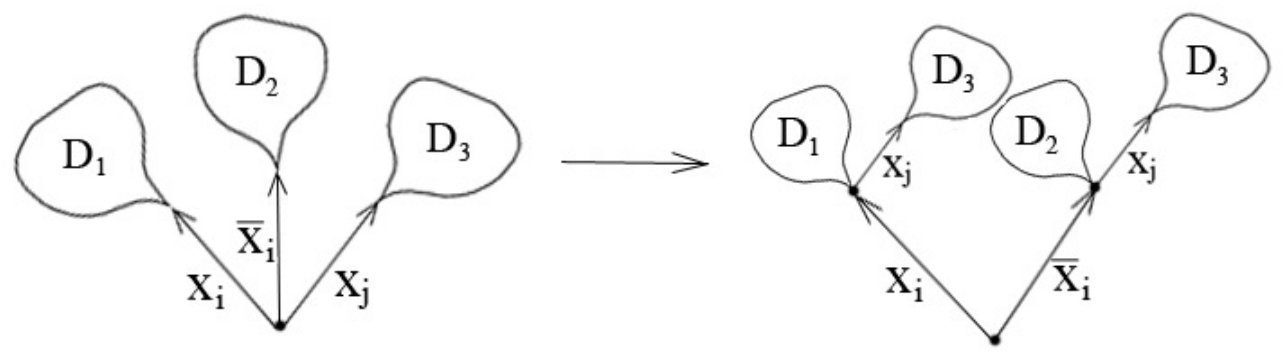

Pис. 1.

сопоставить хотя бы один из этих наборов (для каким-либо образом фиксированного набора индексов $\left.i_{1}, \ldots, i_{s}\right)$, то всего таких функций не больше $2^{2^{n}-2^{n-s}+s+1}\left(\begin{array}{l}n \\ s\end{array}\right)$.

Соответственно получаем, что число функций, для которых $\zeta(f)>s$, не меньше $2^{2^{n}}-2^{2^{n}-2^{n-s}+s+1}\left(\begin{array}{l}n \\ s\end{array}\right)$, что и требовалось доказать.

Теорема 2. Для почти всех булевых функичий $f \in P_{2}^{(n)}$

$$
L(f) \asymp n .
$$

Доказательство. Фиксируем константу $\varepsilon \in(0,1)$ и рассмотрим функции, для которых $\zeta(f)>(1-\varepsilon) n$. Из формулы (2) следует, что сложность таких функций не меньше $(1-\varepsilon) n$. Пользуясь леммой 6 , легко убедиться, что для любого $\varepsilon$ число функций, сложность которых меньше $(1-\varepsilon) n$, есть $o\left(2^{2^{n}}\right)$ при $n \rightarrow \infty$. Следовательно, для почти всех функций $L(f) \geqslant(1-\varepsilon) n$. Однако, мы знаем, что $L(f) \leqslant 2 n-1$. Это означает, что порядок их сложности есть $n$, то есть $L(f) \asymp n$.

Теорема доказана.

\section{7. Асимптотика сложности реализации информационными деревьями для почти всех булевых функций}

Ограничимся теперь только информационными деревьями. Для них мы получим более точную оценку сложности через функции $\zeta_{0}$ и $\zeta_{1}$, что позволит улучшить оценку (1).

Лемма 7. Для сложности реализации произвольной функиии $n$ переменных с помощью информационных деревьев справедлива оиенка

$$
L_{D}(f) \geqslant 2^{-n}\left(N_{0}(f)(2 \zeta(f)-1)+N_{1}(f)(2 \zeta(f)-1)\right)=2 \zeta(f)-1 .
$$

Доказательство. Будем доказывать лемму индукцией по высоте дерева. Для дерева высоты 0 (начальная вершина совпадает с конечной и функция есть тождественная единица) $\zeta_{1}(f)=0, \zeta(f)=0, N_{0}(f)=0, N_{1}(f)=1$. Значение $\zeta_{0}(f)$ не определено, так как функция не может обратиться в 0 ни при какой фиксации переменных (которых вообще нет). 
База индукции выполнена для нуля: $0=L_{D}(f) \geqslant-1$.

Рассмотрим дерево высоты 1 . Если оно реализует константу, то опять получаем, что $\zeta(f)=0$ и неравенство выполнено. Если дерево реализует функцию $x_{1}^{\sigma_{1}} \vee x_{2}^{\sigma_{2}} \vee \ldots \vee x_{s}^{\sigma_{s}}$, то $\zeta_{0}(f)=s, \zeta_{1}(f)=1, \zeta(f)=1$. Мы взяли первые переменные $1, \ldots, s$, но это, очевидно, не существенно.

База индукции выполнена: $s=L_{D}(f) \geqslant 1$. Сделаем некоторые преобразования нашего дерева, которые не изменяют его функциональности и не увеличивают сложности.

Пусть из одной вершины выходят ребра $x_{i}, \bar{x}_{i}, x_{j}^{\sigma_{j}}, \ldots$ Тогда дублируем дерево со стволом $x_{j}^{\sigma_{j}}$ и переместим его на концы ребер $x_{i}$ и $\bar{x}_{i}$ (как показано на рис. 1). Понятно, что функциональность дерева не изменится, потому что любой запрос пройдет ровно по одному из ребер $x_{i}, \bar{x}_{i}$, а дальше будет действовать так же, как будто бы дерево росло из исходной вершины. По той же причине сложность не изменится. Все предикаты на ребрах перемещенного дерева будут вычислены ровно столько же раз, сколько были вычислены до перестановки, потому что произвольный запрос пройдет только по одному из ребер $x_{i}, \bar{x}_{i}$.

Будем считать, что мы применили такое преобразование, если возможно, к каждой вершине графа. Тогда останутся только вершины, из которых выходят либо два ребра $x_{i}, \bar{x}_{i}$ (вершины первого рода), либо несколько ребер $x_{i_{1}}^{\sigma_{i_{1}}}, x_{i_{2}}^{\sigma_{i_{2}}}, \ldots, x_{i_{s}}^{\sigma_{i_{s}}}$, где все $i_{j}-$ различные числа (вершины второго рода).

Если корневая вершина - вершина первого рода, то рассмотрим два дерева $\left(D_{1}\right.$ и $\left.D_{2}\right)$, построенных на концах ребер $x_{i}$ и $\bar{x}_{i}$, и функции, которые они реализуют. Обозначим эти функции $f_{1}$ и $f_{2}$.

Рассмотрим изменение функций $\zeta_{0}$ и $\zeta_{1}$ при переходе от функции $f$ к $f_{1}$ и $f_{2}$. Очевидно, что они не могут уменьшиться больше чем на единицу, так как мы просто фиксируем одну переменную $x_{i}$ единицей, когда идем по ребру $x_{i}$, и нулем, когда идем по ребру $\bar{x}_{i}$. Функция $\zeta$ тоже может уменьшиться максимум на единицу.

Таким образом, получаем, что $\zeta\left(f_{1}\right) \geqslant \zeta(f)-1, \zeta\left(f_{2}\right) \geqslant \zeta(f)-1$. Сложности деревьев $D_{1}$ и $D_{2}$ по предположению индукции не меньше $2 \zeta\left(f_{1}\right)-1$ и $2 \zeta\left(f_{2}\right)-1$ соответственно. Учитывая, что сложности этих деревьев умножаются на $1 / 2$, когда мы их помещаем на концы ребер $x_{i}$ и $\bar{x}_{i}$, получаем, что сложность всего дерева не меньше

$$
\begin{aligned}
2+\frac{1}{2}\left(\left(2 \zeta\left(f_{1}\right)-1\right)+\left(2 \zeta\left(f_{2}\right)-1\right)\right) & \geqslant 2+((2 \zeta(f)-3)+(2 \zeta(f)-3)) \\
& =2 \zeta(f)-1 .
\end{aligned}
$$

Таким образом, случай корневой вершины первого рода разобран.

Теперь пусть корневая вершина - вершина второго рода, то есть из нее выходит несколько ребер, причем все переменные на этих ребрах разные. Из свойств функции $\zeta_{0}$ следует, что число этих ребер не меньше $\zeta_{0}(f)$. Из свойств функции $\zeta_{1}(f)$ следует, что все пути из начальной вершины в конечные имеют длины, не меньшие $\zeta_{1}(f)$. Из свойств функции $\zeta_{0}(f)$ следует также, что существует не меньше $\zeta_{0}(f)$ ребер, выходящих из корня, по которым проходит хотя бы один путь в конечную вершину. В противном случае можно было бы найти такой набор длины, меньшей $\zeta_{0}(f)$, который перекрывает все такие ребра, и следовательно, обращает функцию в ноль.

Итак, подсчитаем сложность дерева $D$. В нем есть хотя бы $\zeta_{0}(f)$ путей длины, не меньшей $\zeta_{1}(f)$. Поэтому его сложность

$$
L(D) \geqslant \zeta_{0}(f)\left(1+2^{-1}+2^{-2}+\ldots+2^{-\zeta_{1}(f)+1}\right)=\zeta_{0}(f)\left(2-2^{-\zeta_{1}(f)+1}\right) .
$$


Осталось сравнить эту величину с $2 \zeta(f)-1$. Для краткости положим $\zeta_{0}=\zeta_{0}(f)$, $\zeta_{1}=\zeta_{1}(f), \zeta=\zeta(f)$. Покажем, что

$$
2 \zeta_{0}-\frac{\zeta_{0}}{2 \zeta_{1}-1} \geqslant 2 \zeta-1
$$

Если $\zeta_{1}>\zeta_{0}$, то очевидно, что $2^{\zeta_{1}-1}>\zeta_{0}$, и

$$
2 \zeta_{0}-\frac{\zeta_{0}}{2 \zeta_{1}-1}>2 \zeta-1 \Longrightarrow L(D) \geqslant 2 \zeta(f)-1
$$

Пусть $\zeta_{1} \leqslant \zeta_{0}$. Тогда $\zeta=\zeta_{1}$ и

$$
\begin{gathered}
2 \zeta_{0}-\frac{\zeta_{0}}{2^{\zeta_{1}-1}} \vee 2 \zeta_{1}-1, \\
\zeta_{0} \vee \frac{2 \zeta_{1}-1}{2-2^{-\zeta_{1}+1}}=\frac{2^{\zeta_{1}} \zeta_{1}-2^{\zeta_{1}-1}}{2^{\zeta_{1}}-1} .
\end{gathered}
$$

Уравнение

$$
\zeta_{1}=\frac{2^{\zeta_{1}} \zeta_{1}-2^{\zeta_{1}-1}}{2^{\zeta_{1}}-1}
$$

имеет только два решения $\zeta_{1}=1$ и $\zeta_{1}=2$, при остальных натуральных $\zeta_{1}$ имеет место неравенство $\zeta_{1}>\left(2^{\zeta_{1}} \zeta_{1}-2^{\zeta_{1}-1}\right) /\left(2^{\zeta_{1}}-1\right)$. Неравенство

$$
\zeta_{0} \geqslant \frac{2^{\zeta_{1}} \zeta_{1}-2^{\zeta_{1}-1}}{2^{\zeta_{1}}-1}
$$

и подавно выполнено. Отсюда получаем, что $L(D) \geqslant 2 \zeta(f)-1$ и в этом случае. Таким образом, лемма доказана.

Теперь найдем асимптотику сложности реализации функции $n$ переменных с помощью информационных деревьев.

Теорема 3. Для почти всех функицй $f \in P_{2}^{(n)}$

$$
L_{D}(f) \sim 2 n-1 .
$$

Доказательство. Фиксируем константу $\varepsilon \in(0,1)$ и рассмотрим функции, для которых $\zeta(f)>(1-\varepsilon) n$. Из формулы (4) следует, что сложность таких функций не меньше $2(1-\varepsilon) n-1$.

Пользуясь леммой 6 , легко убедиться, что для любого $\varepsilon$ число функций, сложность которых меньше $2(1-\varepsilon) n-1$, есть $o\left(2^{2^{n}}\right)$ при $n \rightarrow \infty$. Следовательно, для почти всех функций $L_{D}(f) \geqslant 2(1-\varepsilon) n-1$. Однако, мы знаем, что $L(f) \leqslant 2 n-1$. Это означает, что сложность удовлетворяет неравенству

$$
2(1-\varepsilon) n-1 \leqslant L_{D}(f) \leqslant 2 n-1 .
$$

Это и дает требуемую асимптотику сложности. Таким образом, теорема доказана. 


\section{Список литературы}

1. Гасанов Э. Э., Кудрявцев В. Б., Теория хранения и поиска информации. Физматлит, Москва, 2002.

2. Шуткин Ю. И., Реализация булевых функций с помощью информационных графов. В сб.: Материалы IX международной конференции «Интеллектуальные системы и компьютерные науки». МГУ, Москва, 2006.

3. Лупанов О. Б., Асимптотические оценки сложности управляющих систем. МГУ, Москва, 1984.

Статья поступила 7.12.2006. 